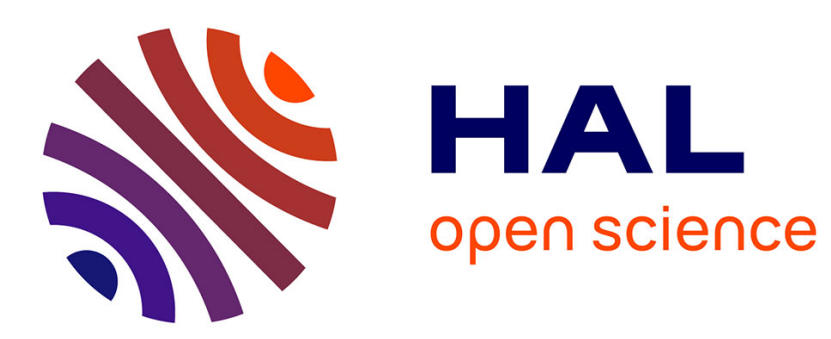

\title{
Sensitivity of Optimal Control Problems Arising from their Hamiltonian Structure
}

\author{
Lamberto Dell'Elce, Daniel J. Scheeres
}

\section{To cite this version:}

Lamberto Dell'Elce, Daniel J. Scheeres. Sensitivity of Optimal Control Problems Arising from their Hamiltonian Structure. Journal of the Astronautical Sciences, In press, 10.1007/s40295-019-00168-1 . hal-02385538

\section{HAL Id: hal-02385538 \\ https://hal.science/hal-02385538}

Submitted on 28 Nov 2019

HAL is a multi-disciplinary open access archive for the deposit and dissemination of scientific research documents, whether they are published or not. The documents may come from teaching and research institutions in France or abroad, or from public or private research centers.
L'archive ouverte pluridisciplinaire HAL, est destinée au dépôt et à la diffusion de documents scientifiques de niveau recherche, publiés ou non, émanant des établissements d'enseignement et de recherche français ou étrangers, des laboratoires publics ou privés. 


\title{
Sensitivity of Optimal Control Problems Arising From Their Hamiltonian Structure
}

\author{
Lamberto Dell'Elce • Daniel J. Scheeres
}

Received: date / Accepted: date

\begin{abstract}
First-order necessary conditions for optimality reveal the Hamiltonian nature of optimal control problems. Regardless of the overwhelming awareness of this result, the implications that it entails have not been fully explored. We discuss how the symplectic structure of optimal control constrains the flow of sub-volumes in the phase space. Special emphasis is devoted to dynamics in the neighborhood of optimal trajectories and insight is gained into how errors in the initial states affect terminal conditions. Specifically, we prove that if the optimal trajectory does not satisfy a particular condition, then there exists a set of variations in the initial states yielding a greater error in norm when mapped to the terminal time through the state transition matrix. We relate this result to the sensitivity problem in solving indirect problems for optimal control.
\end{abstract}

Keywords Hamiltonian systems · Optimal control · Symplectic structures

\section{Introduction}

The symplectic structure of Hamiltonian systems is responsible for fundamental restrictions that apply to the phase flow [Arnold, 1989, de Gosson, 2011]: the conservation of any full-dimensional volume stated by the Liouville's theorem is arguably the most renown among them, whereas other oftentimes-unintuitive constraints impose limitations on how this volume can be deformed or stretched, e.g., Gromov's non-squeezing theorem, Poincaré-Cartan integral invariants, and the Wirtinger inequality [Gromov, 1985, Maruskin et al., 2009].

Understanding these dynamics provides valuable insight into the propagation of uncertainties in Hamiltonian systems [de Gosson and Luef, 2009], with applications to quantum mechanics and astrodynamics [de Gosson, 2009, Hsiao and Scheeres,

\footnotetext{
L. Dell'Elce

Inria Sophia Antipolis Méditerranée

E-mail: lamberto.dell-elce@inria.fr
}

D. J. Scheeres

University of Colorado Boulder, Colorado Center for Astrodynamics Research, 80309 Boulder, Colorado 
2007]. In the context of optimal control, the necessary conditions outlined by the Pontryagin maximum principle reveal the well-known Hamiltonian nature of the problem, where the adjoint variables associated to the enforcement of the equations of movement serve as conjugate momenta [Pontryagin, 1987]. Nonetheless, the implications of this result in the perspective of symplectic structures have not been comprehensively investigated in the literature.

Indirect approaches for optimal control, e.g., shooting method, are of interest because they lend themselves to low-dimensional optimization problems. Nonetheless, their success is often jeopardized by their high sensitivity to variations in the initial states and adjoints, so that direct techniques, e.g., collocation, are used instead. An adequate scaling of the problem can mitigate these effects, but deep understanding of this sensitivity mechanism is missing. The ideal situation for the exploitation of indirect techniques is that any perturbation of the initial conditions is systematically mapped to a 'small' perturbation of the terminal ones.

This work is aimed at assessing how the aforementioned constraints manifest themselves in an optimal control problem and how indirect techniques might benefit from the understanding of this mechanism. Specifically, two questions are tackled: (1) What are the features of symplectic structures when constraints on their terminal states are imposed? (2) What is the sensitivity of optimal trajectories with these constraints with respect to variations in the initial states and adjoints?

After briefly recalling some fundamentals on the Hamiltonial nature of optimal control problems in Section 2, the first question is answered in Section 3, where we focus on Poincaré-Cartan integral invariants. We show that they equal zero when evaluated on the manifold that drives the states to the desired terminal point. The second question is approached in Section 4. Here, we introduce a requirement on the state transition matrix of an optimal trajectory whose fulfillment guarantees that, in the presence of any perturbation of the initial state, the norm of the error on the terminal conditions is smaller than the norm of the perturbation at the initial time. Finally, the outcomes of the paper are illustrated in Section 5 by means of a simple example considering a time optimal planar low-thrust orbital transfer.

\section{Hamiltonian nature of optimal control problems}

Let $\boldsymbol{q} \in \mathbb{R}^{n}$ and $\boldsymbol{u} \in \mathcal{U} \subseteq \mathbb{R}^{m}$ be a set of dynamical and control variables, respectively. The flow of $\boldsymbol{q}$ is governed by the differential equation $\dot{\boldsymbol{q}}=\boldsymbol{f}(\boldsymbol{q}, \boldsymbol{u})$. Consider a maneuver aimed at steering the state vector from some initial conditions, $\boldsymbol{q}_{0}$, to a desired target point, $\boldsymbol{q}_{f}$, after a prescribed time, $t_{f}$. The trajectory is required to minimize the integral of the convex cost function $g(\boldsymbol{x}, \boldsymbol{u})$, so that the optimal control problem is formulated as

$$
\begin{aligned}
& {\left[\boldsymbol{q}^{*}, \boldsymbol{u}^{*}\right]=\arg \left(\min _{\boldsymbol{x}, \boldsymbol{u}} \int_{t_{0}}^{t_{f}} g(\boldsymbol{q}, \boldsymbol{u}) \mathrm{d} t\right) \quad \text { s.t. }:} \\
& \dot{\boldsymbol{q}}=\boldsymbol{f}(\boldsymbol{q}, \boldsymbol{u}), \boldsymbol{u} \in \mathcal{U} \quad \forall t \in\left[t_{0}, t_{f}\right], \\
& \boldsymbol{q}\left(t_{0}\right)=\boldsymbol{q}_{0}, \boldsymbol{q}\left(t_{f}\right)=\boldsymbol{q}_{f} .
\end{aligned}
$$


Denote by $\mathcal{H}^{\prime}$ the pre-Hamiltonian,

$$
\mathcal{H}^{\prime}(\boldsymbol{q}, \boldsymbol{p}, \boldsymbol{u})=-g(\boldsymbol{q}, \boldsymbol{u})+\boldsymbol{p}^{T} \boldsymbol{f}(\boldsymbol{q}, \boldsymbol{u}),
$$

where $\boldsymbol{p} \in \mathbb{R}^{n}$ are the time-varying Lagrange multipliers associated to the constraints $\dot{\boldsymbol{q}}=\boldsymbol{f}(\boldsymbol{q}, \boldsymbol{u})$. In the context of optimal control, $\boldsymbol{p}(t)$ is generally referred to as adjoint vector. Without loss of generality, we assume that both $\boldsymbol{q}$ and $\boldsymbol{p}$ are dimensionless in this paper.

The Pontryagin maximum principle (PMP) [Pontryagin, 1987] outlines necessary conditions that the solution of Problem (1) is required to satisfy. Specifically, the PMP states that the optimal control, $\boldsymbol{u}^{*}$, has to maximize the pre-Hamiltonian, i.e.,

$$
\boldsymbol{u}^{*}(\boldsymbol{q}, \boldsymbol{p})=\arg \left(\max _{\boldsymbol{u}} \mathcal{H}^{\prime}(\boldsymbol{q}, \boldsymbol{p}, \boldsymbol{u})\right),
$$

and that the trajectory of the state and adjoint variables is governed by the Hamilton's equations

$$
\dot{\boldsymbol{q}}=\frac{\partial \mathcal{H}}{\partial \boldsymbol{p}}, \quad \dot{\boldsymbol{p}}=-\frac{\partial \mathcal{H}}{\partial \boldsymbol{q}},
$$

where the Hamiltonian, $\mathcal{H}: \mathbb{R}^{2 n} \rightarrow \mathbb{R}$, is defined as

$$
\mathcal{H}(\boldsymbol{q}, \boldsymbol{p})=\mathcal{H}^{\prime}\left(\boldsymbol{q}, \boldsymbol{p}, \boldsymbol{u}^{*}(\boldsymbol{q}, \boldsymbol{p})\right) .
$$

Indeed, the solution of Problem (1) has also to satisfy the boundary conditions, $\boldsymbol{q}\left(t_{0}\right)=\boldsymbol{q}_{0}$ and $\boldsymbol{q}\left(t_{f}\right)=\boldsymbol{q}_{f}$.

Defining the vector of canonical coordinates $\boldsymbol{x}=\left[\boldsymbol{q}^{T}, \boldsymbol{p}^{T}\right]^{T}$, Eq. (2) can be recast in the compact form

$$
\dot{\boldsymbol{x}}=J \frac{\partial \mathcal{H}}{\partial \boldsymbol{x}},
$$

where

$$
J=\left[\begin{array}{cc}
0_{n} & I_{n} \\
-I_{n} & 0_{n}
\end{array}\right],
$$

and $I_{n}$ and $0_{n}$ denote the $n \times n$ identity and null matrices, respectively,

The solution of Eq. (3) for given initial conditions, $\boldsymbol{x}_{0}$, is denoted by $\phi\left(t, \boldsymbol{x}_{0}\right)$. Hence, in the framework of Problem (1), $\boldsymbol{x}_{0}$ must be such that

$$
\begin{aligned}
{\left[\begin{array}{ll}
I_{n} & 0_{n}
\end{array}\right] \phi\left(0, \boldsymbol{x}_{0}\right) } & =\boldsymbol{q}_{0}, \\
{\left[\begin{array}{ll}
I_{n} & 0_{n}
\end{array}\right] \phi\left(t_{f}, \boldsymbol{x}_{0}\right) } & =\boldsymbol{q}_{f} .
\end{aligned}
$$

In this paper we are interested in the flow of the neighborhood of the optimal trajectory. By perturbing the initial conditions with the $2 n$-dimensional vector $\delta \boldsymbol{x}_{0}$ so that $\boldsymbol{x}(0)=\boldsymbol{x}_{0}+\delta \boldsymbol{x}_{0}$, the resulting trajectory can be expressed as

$$
\phi\left(t, \boldsymbol{x}_{0}+\delta \boldsymbol{x}_{0}\right)=\phi\left(t, \boldsymbol{x}_{0}\right)+\Phi\left(t, \boldsymbol{x}_{0}\right) \delta \boldsymbol{x}_{0}+\mathcal{O}\left(\left\|\delta \boldsymbol{x}_{0}\right\|^{2}\right)
$$

where

$$
\Phi\left(t, \boldsymbol{x}_{0}\right)=\frac{\partial \phi\left(t, \boldsymbol{x}_{0}\right)}{\partial \boldsymbol{x}_{0}}
$$

denotes the state transition matrix (STM). In the remainder of the paper, the initial condition $\boldsymbol{x}_{0}$ in Eq. (5) is understood, so that the shorthand notation $\Phi(t)$ is used. 
The second-order term of Eq. (4) is such that

$$
\lim _{\left\|\delta \boldsymbol{x}_{0}\right\| \rightarrow 0} \frac{\mathcal{O}\left(\left\|\delta \boldsymbol{x}_{0}\right\|^{2}\right)}{\left\|\delta \boldsymbol{x}_{0}\right\|^{2}}<\infty
$$

for a fixed time $t$. Although this quantity may become large and non-negligible when regarded as a function of time for a fixed $\delta \boldsymbol{x}_{0}$, it is neglected in this paper. The evolution of the STM is governed by the initial value problem

$$
\begin{aligned}
& \dot{\Phi}(t)=\left.J \frac{\partial^{2} \mathcal{H}}{\partial \boldsymbol{x}^{2}}\right|_{\boldsymbol{x}^{*}(t)} \Phi(t), \\
& \Phi(0)=I_{2 n} .
\end{aligned}
$$

From Eq. (6), it is straightforward to verify that $\frac{\mathrm{d}}{\mathrm{d} t}\left(\Phi^{T} J \Phi\right)=0$, so that Eq. (6) is an infinitesimal symplectomorfism. For this reason and by noting that $\Phi(0)=I_{2 n \times 2 n} \in \operatorname{Sp}(n)$ is symplectic, it holds that

$$
\Phi(t) \in \operatorname{Sp}(n) \quad \forall t \in\left[t_{0}, t_{f}\right],
$$

where $\operatorname{Sp}(n)=\left\{A \in \mathbb{R}^{2 n \times 2 n}: A^{T} J A=J\right\}$ denotes the group of $2 n$-dimensional real-valued symplectic matrices.

All results discussed in the remainder of the paper are intimately related to the Hamiltonian nature of optimal control disclosed by Eq. (2), which holds true in more general formulations than Problem (1), e.g., non-autonomous system, unspecified maneuvering time, and additional path constraints. However, all developments of this work are built upon Problem (1) to avoid unnecessary notations.

\section{Poincaré-Cartan integral invariants}

Integral invariants are quantities that are defined by means of an integral on an arbitrary region of the configuration manifold and that conserve their value for any symplectomorfism of the region itself.

Let $\Omega\left(t_{0}\right)$ be an arbitrary $2 k$-dimensional sub-volume in the $2 n$-dimensional configuration space at time $t_{0}$. The sum of the signed projections of $\Omega\left(t_{0}\right)$ on all combinations of the symplectic subspaces,

$$
\left\{q_{i_{1}}, p_{i_{1}}, \ldots, q_{i_{k}}, p_{i_{k}}\right\}, \quad 1 \leq i_{1} \leq \ldots \leq i_{k} \leq n
$$

is preserved under any symplectomorfism, which includes the mapping of $\Omega$ under the Hamiltonian flow, i.e.,

$$
\mathcal{I}_{k}(\Omega)=\mathcal{I}_{k}(\phi(t, \Omega)) \quad \forall t \in\left[t_{0}, t_{f}\right]
$$

where

$$
\mathcal{I}_{k}(\Omega)=\sum_{1 \leq i_{1} \leq \ldots \leq i_{k} \leq n} \int_{\Omega} \mathrm{d} q_{i_{1}} \wedge \mathrm{d} p_{i_{1}} \wedge \ldots \wedge \mathrm{d} q_{i_{k}} \wedge \mathrm{d} p_{i_{k}}
$$




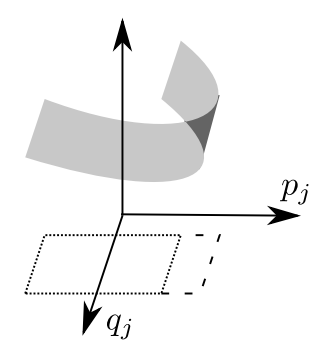

Fig. 1 Projection of a surface on the $j$-th symplectic plane. The dotted region denotes the magnitude of the signed projection. The unsigned projection also includes the dashed region.

and the wedge denotes the exterior product. The quantity $\mathcal{I}_{k}(\Omega)$ is referred to as Poincaré-Cartan invariant of the volume $\Omega$. An intuitive visualization of PoincaréCartan invariants is obtained when a 2-dimensional surface is considered. In this case, the integral becomes

$$
\mathcal{I}_{1}(\Omega)=\sum_{i=1}^{n} \int_{\Omega} \mathrm{d} q_{i} \wedge \mathrm{d} p_{i},
$$

and it consists of the sum of signed projections of the surface $\Omega$ on all symplectic planes $\left(q_{j}, p_{j}\right)$. When subject to the dynamical flow, the surface $\Omega(t)$ may fold. In this case the norm of the unsigned and signed projections can differ from each other, as shown in Figure 1. However, the sum of the signed projections on all symplectic planes $\left(q_{j}, p_{j}\right)$ is preserved.

When a $2 n$-dimensional $\Omega$ is considered, the Poincaré-Cartan invariant states that a non-degenerate volume of the phase space is preserved under any symplectomorfism. This result is also referred to as Liouville's theorem, and it can be alternatively deduced by noting that Hamiltonian flows are divergence-less.

Poincaré-Cartan integrals assume an additional compelling feature in the context of optimal feedback control [Bryson and Ho, 1975], where for any initial state vector, $\boldsymbol{q}_{0}$, the corresponding adjoint vector is tuned such that $\boldsymbol{q}\left(t_{f}\right)=\boldsymbol{q}_{f}$. The solution to this problem satisfies the Hamilton-Jacobi-Bellman equation and it defines an $n$-dimensional manifold on the phase space,

$$
\mathcal{F}=\left\{\boldsymbol{x}_{0} \in \mathbb{R}^{2 n}: \boldsymbol{q}\left(t_{f}\right)=\boldsymbol{q}_{f}\right\},
$$

such that the projection of its volume on the space of the dynamical variables $\boldsymbol{q}$ at time $t_{f}$ is equal to zero. As a consequence, all projections of $\mathcal{F}$ (and of any arbitrary subset of $\mathcal{F}$ ) on the symplectic planes are zero at $t_{f}$, so that the corresponding Poincaré-Cartan invariant is also zero. When mapped back to $t_{0}$, the invariance of the integral imposes a fundamental constraint to the manifold $\mathcal{F}$ : any subvolume with non-zero Poincaré-Cartan integral cannot belong to the manifold $\mathcal{F}$. For example, a surface parallel to the $j$-th symplectic plane cannot reside in $\mathcal{F}$, since all terms of Eq. (7) but the $j$-th one are zero, this giving a non-zero $\mathcal{I}_{1}$. The same holds true for any $2 k$-dimensional volume with its faces parallel to $k$ symplectic planes.

Additional insight is obtained by considering a differential neighborhood of the optimal initial conditions, $\boldsymbol{x}^{*}\left(t_{0}\right)$. Denote by $\Phi_{q q}, \Phi_{q p}, \Phi_{p q}$, and $\Phi_{p p}$ the $n \times n$ 


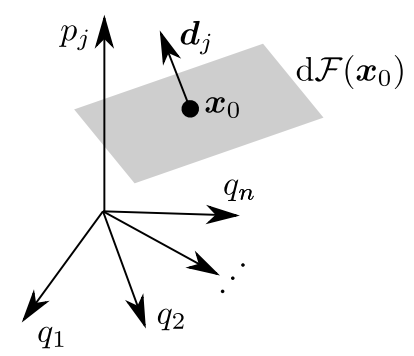

Fig. 2 Optimal feedback control surface (shaded region) and violating direction $\boldsymbol{d}_{j}$.

blocks of the STM such that

$$
\Phi(t)=\left[\begin{array}{ll}
\Phi_{q q}(t) & \Phi_{q p}(t) \\
\Phi_{p q}(t) & \Phi_{p p}(t)
\end{array}\right]
$$

The tangent space of $\mathcal{F}$ at point $\boldsymbol{x}^{*}(0)$ is given by

$$
\mathrm{d} \mathcal{F}=\left\{\delta \boldsymbol{x}_{0}:\left[\Phi_{q q}(t) \Phi_{q p}(t)\right] \delta \boldsymbol{x}_{0}=\mathbf{0}\right\},
$$

and it is illustrated in Figure 2.

Arbitrary subsets of the $n$-dimensional space, $\mathrm{d} \mathcal{F}$, satisfy the aforementioned requirement of zero Poincaré-Cartan integral. In addition, the first $n$ rows of the STM,

$$
\left[\begin{array}{c}
\boldsymbol{d}_{1}^{T} \\
\vdots \\
\boldsymbol{d}_{n}^{T}
\end{array}\right]=\left[\Phi_{q q}(t) \Phi_{q p}(t)\right]
$$

form a complementary basis for $\mathrm{d} \mathcal{F}$,

$$
\mathrm{d} \mathcal{F} \cup \operatorname{span}\left(\boldsymbol{d}_{1}, \ldots, \boldsymbol{d}_{n}\right)=\mathbb{R}^{2 n} .
$$

Any perturbation of the initial conditions with some components in the space spanned by $\boldsymbol{d}_{1}, \ldots, \boldsymbol{d}_{n}$ yields $\delta \boldsymbol{q}\left(t_{f}\right) \neq \mathbf{0}$.

\section{Symplectic eigenskeleton and contracting directions}

Differently of various applications in Hamiltonian mechanics, trajectories of interest in optimal control do not coincide with fixed points of the system, so that the associated STMs are time variant and stability results of linear time invariant systems cannot be exploited to characterize the flow in the neighborhood of the trajectories themselves. For this reason, the developments of this Section are based on the symplectic eigenskeleton introduced in [Maruskin et al., 2009] instead.

Denote by $\Phi(t)$ the STM associated to the trajectory $\phi\left(t, \boldsymbol{x}_{0}\right)$. The symplectic eigenskeleton of the transformation $\phi\left(t, \boldsymbol{x}_{0}\right)$ is defined as the eigenbasis of the STM's Gramian, $\Phi^{T}(t) \Phi(t)$. The eigenvectors of $\Phi^{T}(t) \Phi(t)$ occur in pairs, $\left(\boldsymbol{\xi}_{\boldsymbol{j}}, \boldsymbol{\nu}_{j}\right)$ such that

$$
\boldsymbol{\nu}_{j}=J \boldsymbol{\xi}_{j}, \quad j=1, \ldots, n .
$$


In addition, the eigenvalues associated to $\boldsymbol{\xi}_{j}$ and $\boldsymbol{\nu}_{j}$ are $\lambda_{j}$ and $\lambda_{j}^{-1}$, respectively, with $0 \leq \lambda_{j} \leq 1$. The vectors $\left\{\boldsymbol{\xi}_{1}, \ldots, \boldsymbol{\xi}_{n}, \boldsymbol{\nu}_{1}, \ldots, \boldsymbol{\nu}_{n}\right\}$ form an orthonormal basis, so that, by defining the matrices $\Xi=\left[\boldsymbol{\xi}_{1}, \ldots, \boldsymbol{\xi}_{n}\right]$ and $N=\left[\boldsymbol{\nu}_{1}, \ldots, \boldsymbol{\nu}_{n}\right]$, it holds

$$
\left[\Xi \begin{array}{ll}
\Xi & N
\end{array}\left[\begin{array}{l}
\Xi^{T} \\
N^{T}
\end{array}\right]=\mathcal{I}_{2 n}\right.
$$

The symplectic eigenskeleton and the eigenvalues $\lambda_{j}$ provide insight into how errors in the initial conditions are mapped to final states. For example, consider a small sphere in the phase space encircling $\boldsymbol{x}_{0}$. After applying the Hamiltonian flow, the sphere is squeezed in the directions $\Phi(t) \boldsymbol{\xi}_{j}$ and stretched in the directions $\Phi(t) \boldsymbol{\nu}_{j}$. The amount of stretch, $\sqrt{\lambda_{j}}$, is closely related to the finite-time Lyapunov exponents.

The symplectic eigenskeleton, allow to decompose the STM in the form

$$
\Phi=R\left[\begin{array}{cc}
\Lambda^{1 / 2} & 0_{n} \\
0_{n} & \Lambda^{-1 / 2}
\end{array}\right]\left[\begin{array}{l}
\Xi^{T} \\
N^{T}
\end{array}\right]
$$

where $\Lambda=\operatorname{diag}\left(\lambda_{1}, \ldots \lambda_{n}\right)$ and $R$ is a symplectic rotation, i.e.,

$$
R \in(\mathrm{Sp}(n) \cap \mathrm{SO}(2 n)) .
$$

Here, $\mathrm{SO}(2 n)=\left\{R \in \mathbb{R}^{2 n \times 2 n}: R^{T} R=\mathcal{I}_{2 n}\right\}$ denotes the special orthogonal group in dimension $2 n$.

To show that Eq. 10 holds true, we note that the last two factors of Eq. (9) are invertible and, by using Eq. (8) and the definition of $\Lambda$, the matrix $R$ is given by

$$
R=\Phi[\Xi N]\left[\begin{array}{cc}
\Lambda^{-1 / 2} & 0_{n} \\
0_{n} & \Lambda^{1 / 2}
\end{array}\right] .
$$

The right-hand term of Eq. (11) consists of the product of three symplectic matrices, so that $R$ is also symplectic. In addition, the matrix $\Phi^{T} \Phi$ can be expressed by means of both Eq. (9) and its spectral decomposition, which gives

$$
\left[\begin{array}{ll}
\Xi & N
\end{array}\left[\begin{array}{cc}
\Lambda^{1 / 2} & 0_{n} \\
0_{n} & \Lambda^{-1 / 2}
\end{array}\right] R^{T} R\left[\begin{array}{cc}
\Lambda^{1 / 2} & 0_{n} \\
0_{n} & \Lambda^{-1 / 2}
\end{array}\right]\left[\begin{array}{l}
\Xi^{T} \\
N^{T}
\end{array}\right]=\left[\Xi \begin{array}{ll}
\Xi & N
\end{array}\right]\left[\begin{array}{cc}
\Lambda & 0_{n} \\
0_{n} & \Lambda^{-1}
\end{array}\right]\left[\begin{array}{l}
\Xi^{T} \\
N^{T}
\end{array}\right] .\right.
$$

Pre- and post-multiplying Eq.(12) by

$$
\left[\begin{array}{cc}
\Lambda^{-1 / 2} & 0_{n} \\
0_{n} & \Lambda^{1 / 2}
\end{array}\right]\left[\begin{array}{l}
\Xi^{T} \\
N^{T}
\end{array}\right] \text { and }[\Xi N]\left[\begin{array}{cc}
\Lambda^{-1 / 2} & 0_{n} \\
0_{n} & \Lambda^{1 / 2}
\end{array}\right]
$$

respectively, yields

$$
R^{T} R=\mathcal{I}_{2 n},
$$

which shows that $R$ is also a $2 n$-dimensional orthogonal matrix.

As mentioned in the introduction of the paper, indirect techniques would benefit if any perturbation of the initial conditions were systematically mapped to a 'small' perturbation of the terminal states. Because $q_{0}$ is given, this requirement can be formulated as

$$
\left\|\delta \boldsymbol{q}\left(t_{f}\right)\right\| \leq\|\delta \boldsymbol{p}(0)\| \quad \forall \delta \boldsymbol{p}(0) \in \mathbb{R}^{n},
$$


The more restrictive notion of omni-directional contraction is now introduced. Specifically, an STM is referred to as an omni-directional contraction if

$$
\left\|\delta \boldsymbol{q}\left(t_{f}\right)\right\| \leq\left\|\delta \boldsymbol{x}_{0}\right\| \quad \forall \delta \boldsymbol{x}_{0} \in \mathbb{R}^{2 n}
$$

where

$$
\left.\left\|\delta \boldsymbol{q}\left(t_{f}\right)\right\|=\|\left[\mathcal{I}_{n} 0_{n}\right] \Phi\left(t_{f}\right) \delta \boldsymbol{x}_{0}\right) \| .
$$

The concept of contraction helps understanding how a favorable formulation of the problem relates to the violating directions defined in Section 3.

Below, we establish a necessary and sufficient condition for an STM to be an omni-directional contraction and, consequently, we provide a sufficient condition for the satisfaction of Eq. (13). For this purpose, we recall that any symplectic rotation, $R$, can be written in the form

$$
R=\left[\begin{array}{cc}
A & B \\
-B & A
\end{array}\right]
$$

where $A$ and $B$ are $n \times n$ matrices such that

$$
\begin{aligned}
& A^{T} A+B^{T} B=\mathcal{I}_{n}, \\
& A^{T} B-B^{T} A=0_{n} .
\end{aligned}
$$

Theorem 1 Consider an STM and its decomposition according to Eq. (9). Let A and $B$ be the $n$-dimensional block matrices of the symplectic rotation as defined in Eq. (14). Then, the STM is an omni-directional contraction if and only if

$$
C:=\mathcal{I}_{n}-\left(A \Lambda A^{T}+B \Lambda^{-1} B^{T}\right) \succeq 0
$$

In the remainder, $C$ is referred to as contracting matrix.

Proof Equation (15) can be proven by expressing the norm of $\delta \boldsymbol{q}\left(t_{f}\right)$ as

$$
\begin{aligned}
\left\|\delta \boldsymbol{q}\left(t_{f}\right)\right\|^{2} & =\delta \boldsymbol{q}\left(t_{f}\right)^{T} \delta \boldsymbol{q}\left(t_{f}\right) \\
& =\delta \boldsymbol{x}_{0}^{T}\left[\begin{array}{l}
\Phi_{q q}^{T}(t) \\
\Phi_{q p}^{T}(t)
\end{array}\right]\left[\Phi_{q q}(t) \Phi_{q p}(t)\right] \delta \boldsymbol{x}_{0}
\end{aligned}
$$

Hence, the difference $\left\|\delta \boldsymbol{x}_{0}\right\|^{2}-\left\|\delta \boldsymbol{q}\left(t_{f}\right)\right\|^{2}$ is nonnegative if

$$
\left[\begin{array}{l}
\Phi_{q q}^{T}(t) \\
\Phi_{q p}^{T}(t)
\end{array}\right]\left[\Phi_{q q}(t) \Phi_{q p}(t)\right] \succeq \mathcal{I}_{2 n} \quad \Rightarrow \quad\left[\Phi_{q q}(t) \Phi_{q p}(t)\right]\left[\begin{array}{l}
\Phi_{q q}^{T}(t) \\
\Phi_{q p}^{T}(t)
\end{array}\right] \succeq \mathcal{I}_{n}
$$

because of the properties of singular values. Substituting Eqs. (9) and (11) into Eq. (16) yields the requirement on the positive definiteness of the contracting matrix defined in Eq. (15). 
We emphasize that Theorem 1 provides a necessary and sufficient condition, so that if the STM is not an omni-directional contraction, then there exists at least one direction in the phase space, $\delta \boldsymbol{x}_{0}$, such that its mapping through the STM yields a perturbation $\delta \boldsymbol{q}\left(t_{f}\right)$ larger in norm than $\delta \boldsymbol{x}_{0}$, i.e.,

$$
\left\{\delta \boldsymbol{x}_{0}:\left\|\delta \boldsymbol{q}\left(t_{f}\right)\right\|>\left\|\delta \boldsymbol{x}_{0}\right\|\right\} \neq \emptyset .
$$

Hence, if Eq. (15) does not hold true, as it is most often the case in real-life applications, the effectiveness of indirect techniques often deteriorates for large $t_{f}$ owing to the generally-exponential increase of the eigenvalues $\lambda_{j}^{-1}$.

To gain insight into the concept of omni-directional contraction, consider the case where $B=0_{n}$. In this case, $A^{T} A=\mathcal{I}_{n}$, so that Eq. (15) simplifies to $\mathcal{I}_{n} \succeq \Lambda$, which holds true because $\lambda_{j} \leq 1, j=1, \ldots, n$. In this case, all the violating directions $\boldsymbol{d}_{j}$ defined in Section 3 can be written as a linear combination of the contracting directions $\boldsymbol{\xi}_{j}$. When $B \neq 0_{n}$, it is required that the contribution of the expanding directions $\boldsymbol{\nu}_{j}$ is small enough to guarantee the satisfaction of Eq. (15).

However, being an omni-directional contraction is neither a property of the system nor of the STM, and the eigenvalues of the contracting matrix are prone to be modified if a canonical change of variables is performed. Block-diagonal transformations are particularly of interest because they do not mix states and adjoints. In addition, it is straightforward to show that these eigenvalues are invariant to symplectic rotations with null off-diagonal blocks, so that the canonical transformation should either include some stretching, i.e., scaling, and/or it does not have to preserve angles. This conjecture is further argued in Section 5.

\section{Example}

We consider the extremal flow of a planar time-optimal maneuver in the two-body problem. The Hamiltonian of the problem in equinoctial elements is

$$
\mathcal{H}=p_{4} \sqrt{\frac{\mu}{q_{1}^{3}}}\left(1+q_{2} \cos q_{4}+q_{3} \sin q_{4}\right)^{2}+F \frac{q_{1}^{3}}{\mu} \sqrt{\sum_{i=1}^{2}\left(\boldsymbol{h}_{i}(\boldsymbol{q}) \cdot \boldsymbol{p}\right)^{2}}
$$

where $\mu$ and $F$ denote the gravitational parameter and the maximum thrust-tomass ratio, respectively, and

$$
\boldsymbol{h}_{1}(\boldsymbol{q})=\left\{\begin{array}{c}
0 \\
\sin q_{4} \\
\cos q_{4} \\
0
\end{array}\right\} \quad \text { and } \quad \boldsymbol{h}_{2}(\boldsymbol{q})=\left\{\begin{array}{c}
\frac{2 p_{1} q_{1}}{1+q_{2} \cos q_{4}+q_{3} \sin q_{4}} \\
\cos q_{4}+\frac{q_{2}+\cos q_{4}}{1+q_{2} \cos q_{4}+q_{3} \sin q_{4}} \\
\sin q_{4}+\frac{q_{3}+\sin q_{4}}{1+q_{2} \cos q_{4}+q_{3} \sin q_{4}} \\
0
\end{array}\right\} .
$$

Table 1 lists the numerical values of the parameters and the initial conditions of the trajectory.

Figure 3(a) depicts the eigenvalues of the contracting matrix for the unscaled problem, i.e., the length of the semi-latus rectum is measured in kilometers. The stretching of two violating directions grows up quickly at the very beginning of the maneuver and the condition outlined in Eq. (15) is never attained. 
Table 1 Parameters and initial conditions for the simulations.

\begin{tabular}{lr}
\hline \hline Parameter & \\
Gravitational parameter, $\mu$ & $3.986 \cdot 10^{9} \mathrm{~km}^{3} \mathrm{~s}^{-2}$ \\
Thrust-to-mass ratio, $F$ & $10^{-4} \mathrm{~N} \mathrm{~kg}^{-1}$ \\
Initial conditions & \\
Semi-latus rectum, $q_{1}$ & $10^{4} \mathrm{~km}$ \\
x-component of the eccentricity vector, $q_{2}$ & 0.75 \\
y-component of the eccentricity vector, $q_{3}$ & 0 \\
True longitude, $q_{4}$ & $0 \mathrm{rad}$ \\
Adjoint vector, $\boldsymbol{p}$ & {$[0,1,0,0]$} \\
\hline \hline
\end{tabular}

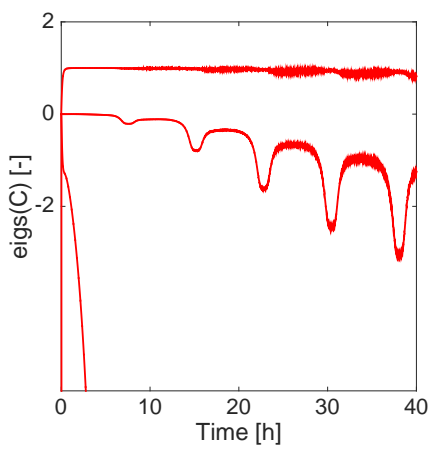

(a) Bad scaling

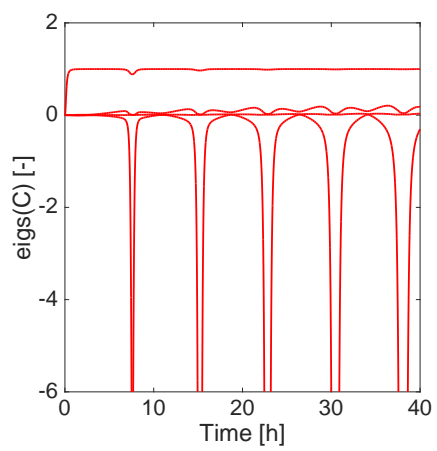

(b) Good scaling

Fig. 3 Eigenvalues of the contracting matrix as a function of time.

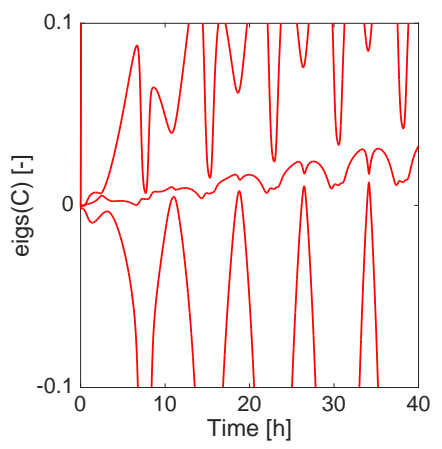

Fig. 4 Eigenvalues of the contracting matrix as a function of time. Zoomed view with the good scaling.

Rescaling the problem consist of applying the canonical transformation

$$
\hat{\boldsymbol{x}}=\left[\begin{array}{cc}
\boldsymbol{s} & 0_{n \times n} \\
0_{n \times n} & \boldsymbol{s}^{-1}
\end{array}\right] \boldsymbol{x},
$$

where $s$ is a diagonal matrix. Figure 3(b) and the close-up view of Figure 4 depict the eigenvalues of the contracting matrix of the rescaled problem with $\operatorname{diag}(s)=\left[20 \cdot 10^{3} \mathrm{~km}, 1,1,100 \mathrm{rad}\right]$. At the beginning of the maneuver, the STM 


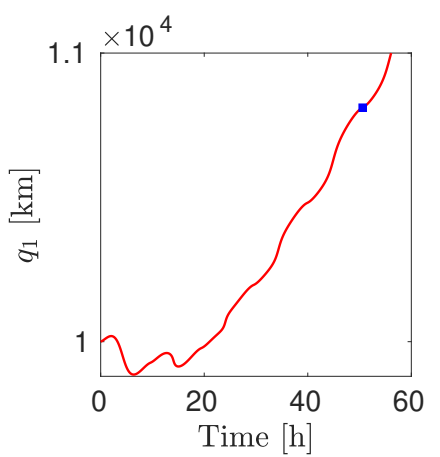

(a)

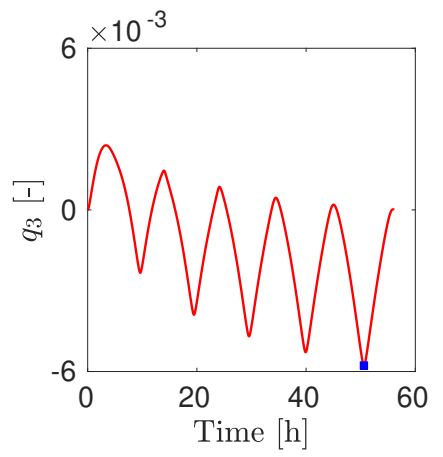

(c)

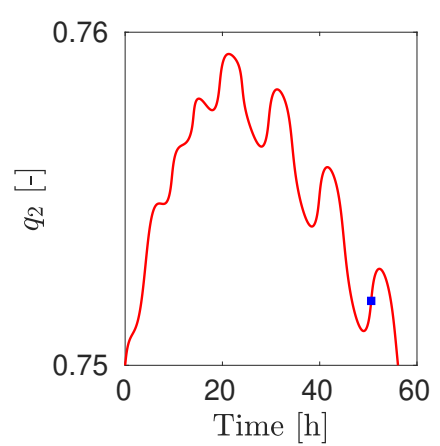

(b)

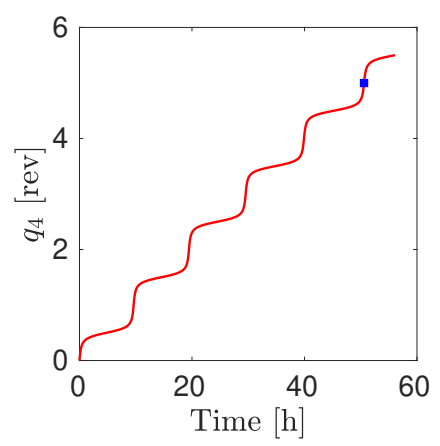

(d)

Fig. 5 Solution of the shooting problem (state trajectory). Blue squares indicate terminal conditions at perigee.

is nearly an omni-directional contraction over several hours. This suggests that the re-scaled problem exhibit less sensitivity with respect to the non-scaled one. In addition, the positive definiteness of the contracting matrix is also attained whenever the satellite is close to the apogee of the orbit. Hence, solving a twopoint boundary value problem with a shooting algorithm is supposedly easier if the desired terminal conditions of the maneuver are close to the apogee. This information could be also exploited for choosing when to update the solution during its execution. As an example, Figure 5 depicts the trajectory obtained as a solution of a two-point boundary value problem with terminal conditions equal to $\mathbf{q}\left(t_{f}\right)=\left[11^{4} \mathrm{~km}, 0.75,0, n \pi\right]$ for an arbitrary odd $n$ (i.e., the terminal state is at apogee). Initial conditions and guess of the initial adjoints are provided in Table 1 . The guess of the maneuvering time is set to 50 hours $^{1}$. Then, a second set of terminal conditions is chosen on this trajectory such that the final anomaly occurs at perigee (blue squares in Figure 5), and the guess of the maneuvering time is re-scaled accordingly. Hence, the initial adjoint vector solution of the two problems is the same. However, convergence is faster in the first case, as shown in Figure 6. Indeed, a thorough and exhaustive investigation is mandatory to confirm

1 For repeatability purposes, the shooting problem is solved by means of the fsolve function of MATLAB using default settings 


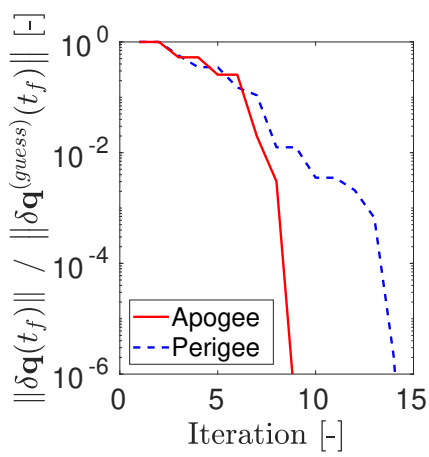

Fig. 6 Convergence of the two-point boundary value problems with terminal conditions at apogee and perigee.

this intuition. We also note that the solver is not able to converge to a feasible solution if states are not scaled.

\section{Conclusion}

This paper discussed fundamental constraints on the flow of dynamical systems in optimal control. The symplectic structure of the problem and the imposition of terminal constraints resulted in findings that are not usual in other fields of Hamiltonian mechanics.

Useful resources are readily available by computing the STM of optimal trajectories. First, we identified the local directions orthogonal to the manifold returning the exact terminal conditions. Any perturbation of the initial states with components in this subspace returns a non-zero error in the terminal conditions. Second, after introducing the notion of omni-directional contraction, we derived necessary and sufficient conditions guaranteeing that any perturbation of the initial states is mapped to a 'small' perturbation of the terminal conditions. This condition exposes one mechanism to which an optimal control problem has high sensitivity to errors in the initial adjoint variables.

Acknowledgements This work was partially supported by the Belgian National Fund for Scientific Research (FNRS) and by the Air Force Office of Scientific Research (AFOSR).

On behalf of all authors, the corresponding author states that there is no conflict of interest.

\section{References}

[Arnold, 1989] Arnold, V. I. (1989). Mathematical Methods of Classical Mechanics. Springer New York.

[Bryson and Ho, 1975] Bryson, A. E. and Ho, Y.-C. (1975). Applied optimal control: optimization, estimation and control. CRC Press.

[de Gosson and Luef, 2009] de Gosson, M. and Luef, F. (2009). Symplectic capacities and the geometry of uncertainty: The irruption of symplectic topology in classical and quantum mechanics. Physics Reports, 484(5):131-179. 
[de Gosson, 2009] de Gosson, M. A. (2009). The symplectic camel and the uncertainty principle: The tip of an iceberg? Foundations of Physics, 39(2):194-214.

[de Gosson, 2011] de Gosson, M. A. (2011). Symplectic Methods in Harmonic Analysis and in Mathematical Physics. Springer Science + Business Media.

[Gromov, 1985] Gromov, M. (1985). Pseudo holomorphic curves in symplectic manifolds. Inventiones Mathematicae, 82(2):307-347.

[Hsiao and Scheeres, 2007] Hsiao, F.-Y. and Scheeres, D. J. (2007). Fundamental constraints on uncertainty evolution in hamiltonian systems. IEEE Transactions on Automatic Control, 52(4):686-691.

[Maruskin et al., 2009] Maruskin, J. M., Scheeres, D. J., and Bloch, A. M. (2009). Dynamics of symplectic subvolumes. SIAM Journal on Applied Dynamical Systems, 8(1):180-201.

[Pontryagin, 1987] Pontryagin, L. S. (1987). Mathematical Theory of Optimal Processes. Classics of Soviet Mathematics. Taylor \& Francis. 